\title{
The Pacific Rim Library: A Surprising Pearl
}

\begin{abstract}
The Pacific Rim Library (PRL) is an initiative of the Pacific Rim Digital Library Association (PRDLA). The project began in 2006 using the OAI-PMH paradigm and now holds over 300,000 records harvested from OAI data provider libraries around the Pacific. PRL's goal is to enable the sharing of digital collections amongst PRDLA members and the world, but greater unexpected benefits have been discovered. Through mirroring their metadata, PRL increases the chance that their data will be discovered in Google and other general search engines. With its many disparate collections, PRL is not a repository for traditional information discovery and retrieval. Initially users will bounce from a Google hit, to the PRL metadata record in Hong Kong, and then begin an intensive search on the original site which hosts the full digital object, in Vancouver, Honolulu, Wuhan, Singapore, or other PRDLA member location. Serials Review 2009; xx:xxx-xxx. (c) 2009 Published by Elsevier Inc.
\end{abstract}

Keywords: OAI; Pacific Rim Library; Pacific Rim Digital Library Association; PRDLA; PRL; Information discovery and retrieval; Indexing; Metadata; Deep Web

\section{Introduction}

The Pacific Rim Digital Library Association (PRDLA) was formed in 1997 by fourteen libraries in the Pacific region. Now thirty-one libraries strong, the goal of this group is to "improve access to scholarly research materials through cooperative ventures." ${ }^{1,2}$ Over the years the association has sponsored many such endeavors. A task force was established in 2004 to study the best means for sharing PRDLA digital collections amongst themselves and the world. In its 2005 report, the task force recommended creating a Web-based Open Archives Initiative (OAI) ${ }^{3}$ and determined that establishing a Service Provider would be the quickest, easiest, and most effective way of accomplishing this goal.

\section{Open Archives Initiative Model}

In the OAI paradigm, there can be many geographically or categorically disparate Data Providers hosting their own local repositories, with digital objects of full-text items or other files. Each Data Provider will expose the metadata on these items; a Service Provider can then harvest, importing the metadata (and not the digital object) into a new repository. The Service Provider will apply a search engine on this new repository. Users can then do one search across all metadata of the several Data Providers. Upon choosing one record and clicking, the user leaves the Service Provider's repository and arrives on a page in one of the local Data Provider's repositories.

\section{Developments and Refinements}

In 2006, a new task force was funded by PRDLA to create an OAI pilot repository at The University of Hong Kong (HKU). The

Palmer is Scholarly Communications Head, HKU Libraries, The University of Hong Kong, Hong Kong; e-mail: dtpalmer@hku.hk. working title of this repository was the "PRDLA Archive," which 46 recently became "Pacific Rim Library" or "PRL" for short. (Here- 47 after, "PRL" will be used, although the name change has still not 48 been enacted.) PRDLA decided that PRL would harvest OAI 49 metadata from at least one locally created digital collection at 50 each of the PRDLA member libraries. HKU became the OAI Service 51 Provider, harvesting data from the many OAI Data Providers. 52 Several PRDLA libraries then implemented the OAI protocol for 53 metadata harvesting (OAI-PMH) for the first time. The PRL OAI 54 data providers created XML compatible, UTF- 8 compliant meta- 55 data using the simplest Dublin Core (DC $)^{4}$ schema: "oai_dc" 56 consisting of fifteen unqualified data elements. At that time, HKU 57 had begun to use DSpace for its institutional repository, and thus, 58 created a second instance of DSpace to be the OAI Service Provider 59 for harvesting and hosting the metadata from the many PRL OAI 60 Data Providers. Each PRDLA member became a "community" in the 61 DSpace paradigm (Fig. 1).

DSpace worked well when there were few records. After more 63 PRDLA member repositories were harvested, however, and record 64 numbers reached 300,000, the response time slowed considerably. 65 We also found problems in OAI harvesting and updating. We then 66 found a replacement in CDS Invenio, ${ }^{5}$ another open source 67 software, developed by CERN. Invenio promises fast searching 68 across a repository of up to $1.5 \mathrm{M}$ records at one time. CDS Invenio 69 was easier to customize for OAI harvesting and provided several 70 out-of-the-box interfaces in different languages and scripts. Our 71 developer also contributed to the code base by making new 72 translations for traditional and simplified Chinese as shown in the 73 following illustration (Fig. 2). ${ }^{6}$

In order to increase the value of PRL, we changed the oai_dc 75 schema used among the libraries, to include one more qualification 76 on Identifier, that of an "identifier thumbnail." This identifier 77 allows for the harvesting and storage of thumbnail images along 78 with the usual bibliographic details. 


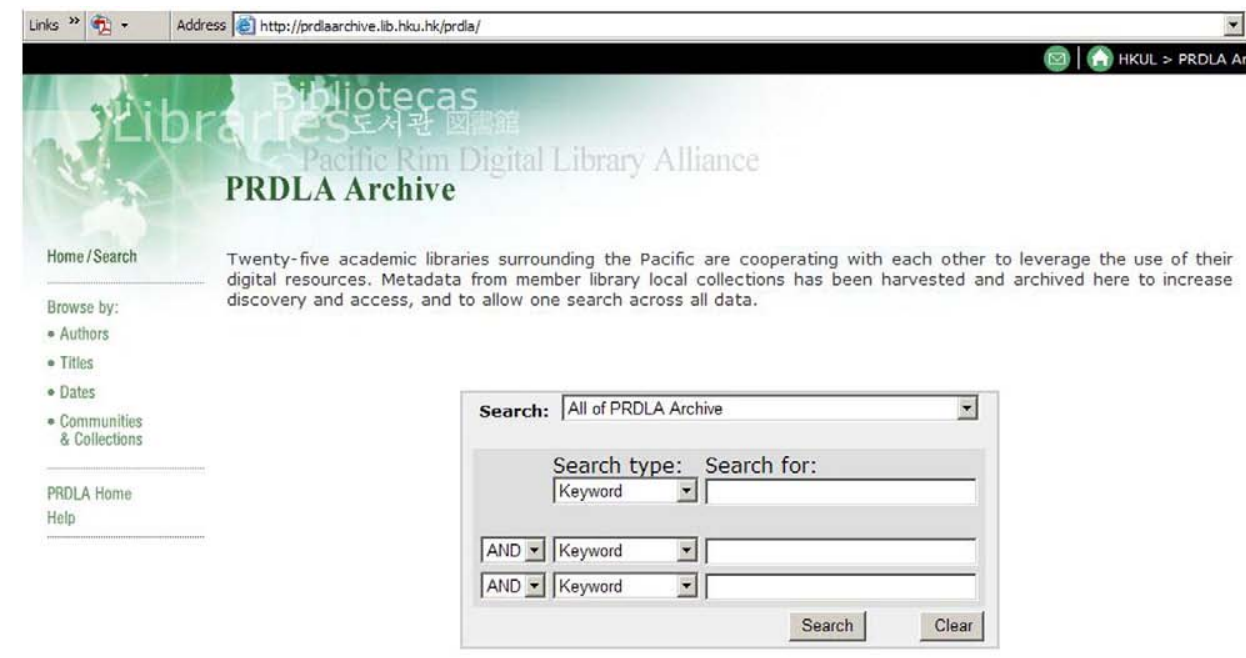

Figure 1. PRDLA archive in DSpace (2006).

The 2007 Berkeley PRDLA meeting pronounced this repository fit for purpose and asked that it go into "production." We removed the password access and specifically invited external robots and crawlers inside the system by creating sitemaps for the major search engines to index content. ${ }^{7}$ We created records for PRL in the following major $\mathrm{OAI}-\mathrm{PMH}$ registries and repositories:

- Open Archives list of registered OAI repositories: http:// www.openarchives.org/Register/BrowseSites

- OAI registry at University of Illinois at Urbana-Champaign: http://gita.grainger.uiuc.edu/registry/

- Celestial OAI registry: http://celestial.eprints.org

- Registry of Open Access Repositories: http://roar.eprints.org/

- Directory of Open Access Repositories - OpenDOAR: http:// www.opendoar.org/

\section{The Purpose of PRL}

Although the membership of PRDLA was generally pleased with 95 the progress of PRL, at the 2007 meeting some members expressed 96 uneasiness at the disparate nature of the collections hosted in PRL. 97 Traditionally, databases are created and chosen for searching 98 because of a selection process in the creation of these databases 99 that strives to include as much relevant content in a given subject 100 as possible, and which, therefore, excludes material beyond the 101 scope of that subject. Although there are many collections in PRL 102 that are unquestionably relevant to the Pacific area, such as the 103 "Sea of Korea Map Collection" and the "Hawaiian Photo Album," 104 there are also several that do not have direct relevance to the 105 Pacific area, such as "The Automobile Club of Southern California." 106

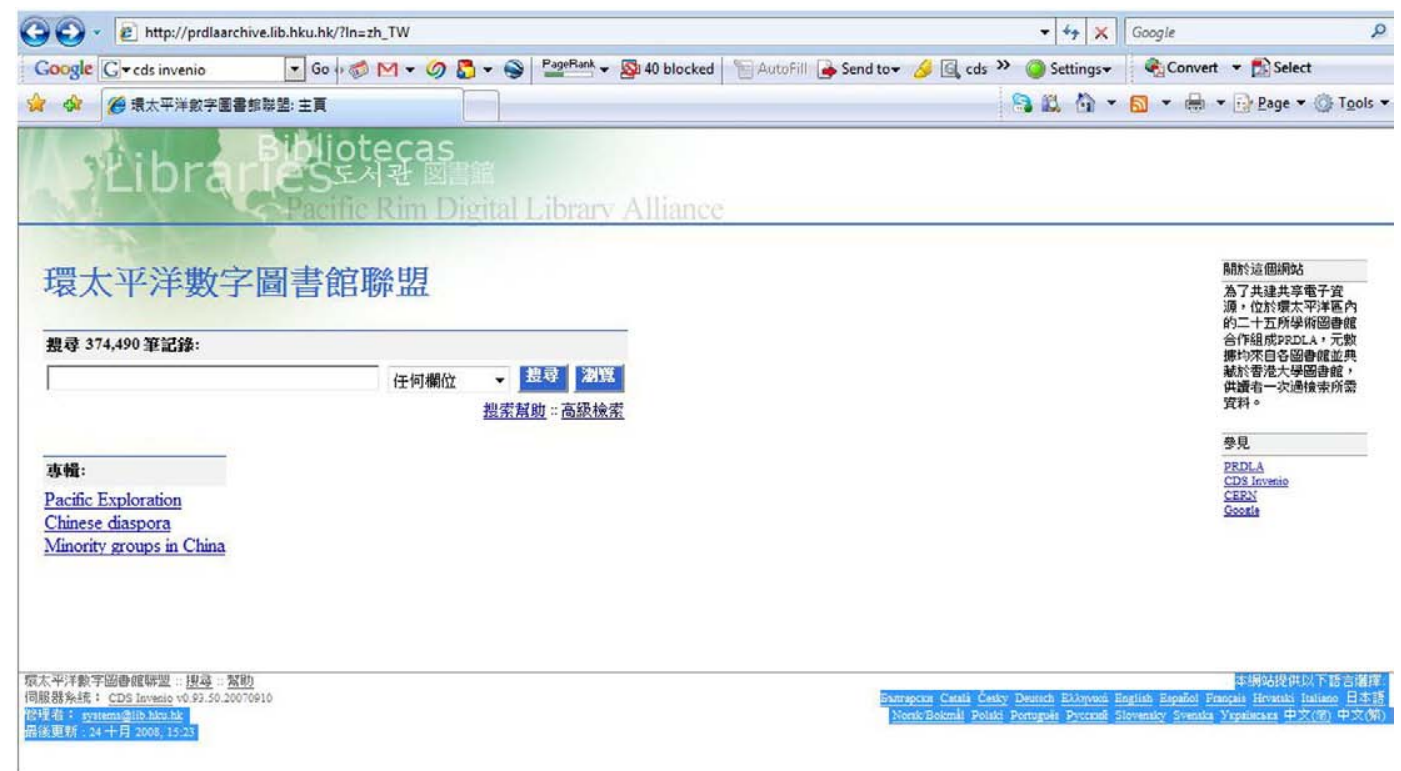

Figure 2. PRDLA archive in CDS Invenio; simplified Chinese interface. 


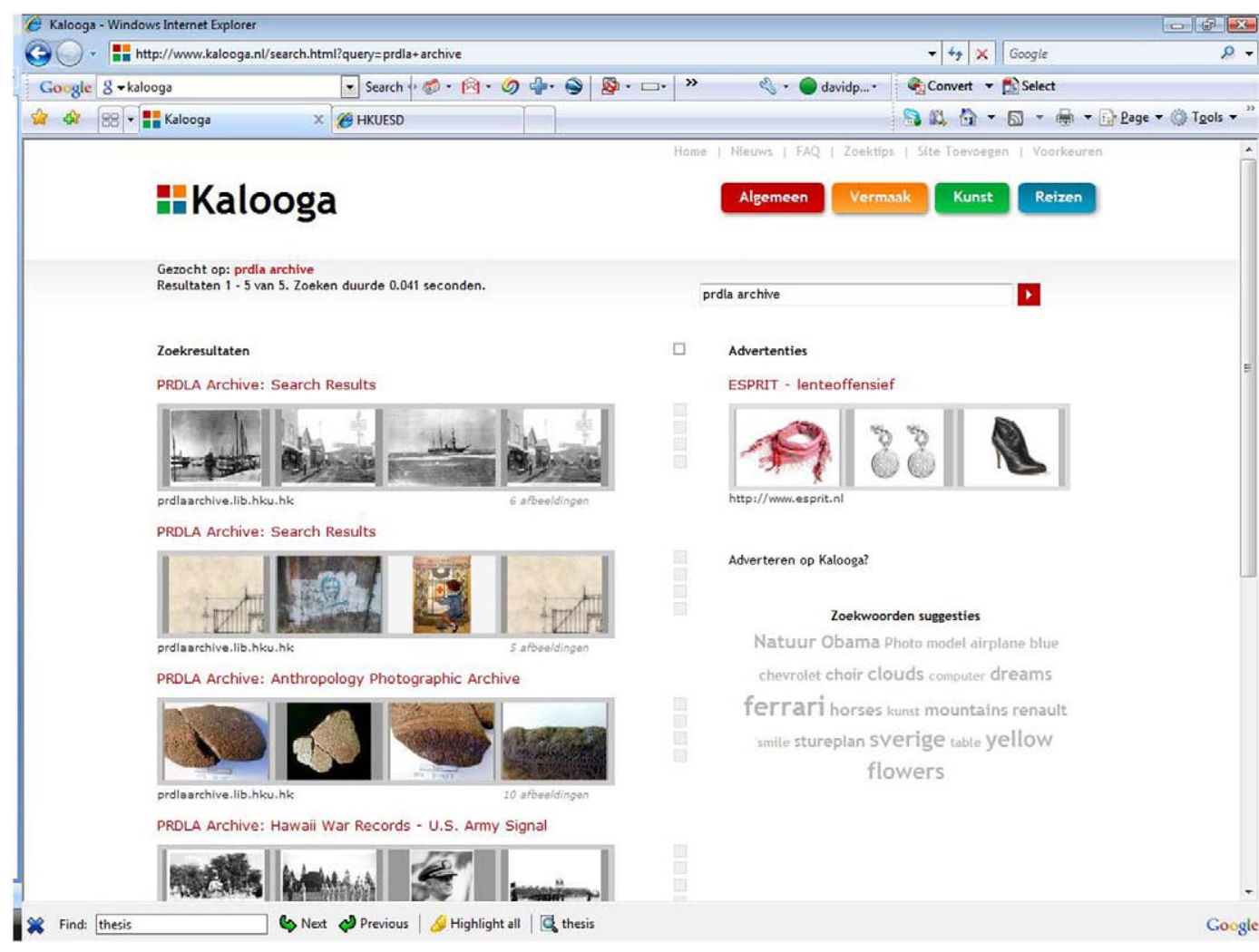

Figure 3. PRL thumbnails in Kalooga.

PRDLA charged a new task force to survey the membership and propose scope and direction for PRL. This task force reported its findings at the 2008 Singapore meeting and made the following recommendations:

1. Change the name to "Pacific Rim Library" with the acronym "PRL," pronounced as "Pearl" (as in "pearl of the orient") to more accurately represent the content of this repository.

2. That the contents of PRL be about the Pacific Rim areas and that the purpose of PRL is to provide people with a way of finding material about the peoples, cultures, history, etc. of the Pacific Rim.

3. That PRDLA should invite others with Pacific Rim-related content to allow harvesting into PRL, and that this invitation be extended to anyone with relevant content, within or external to the PRDLA membership.

\section{Unexpected Pearls}

The PRL Technical Committee also presented a report at this meeting that described its findings on the usage statistics of PRL. These statistics showed high activity by all of the major search bots, including Googlebot, Yahoo Slurp, Cuil's Twiceler, BaiduSpider, etc. One top referring site was a Wikipedia entry about James Wong (黃湛森), a recently deceased Cantopop singer and songwriter from Hong Kong. At that time, the entry hyperlinked to the record in PRL, which, in turn, hyperlinked to his full-text $\mathrm{PhD}$ thesis in the HKU Libraries. One top target identified in PRL was the War Poster Collection of the University of Washington. Thumbnail images provided by this collection in PRL are very attractive to Kalooga, and other major projects seeking thumbnail images (Fig. 3).

\section{Search Engine Results}

Comparing searches in Google and Yahoo on a known PRL title, with 137 the original host providing the metadata, gives interesting results. A 138 Google search in the HKU network on "Lise's Lunchwagon" shows 139 only two hosts, the originating collection at the University of 140 Hawaii-Manoa and PRL (the UH-M record appears above PRL, as 141 expected, because they are the original provider); however, a search 142 on "Hoo Hoo House," a record from the University of Washington, 143

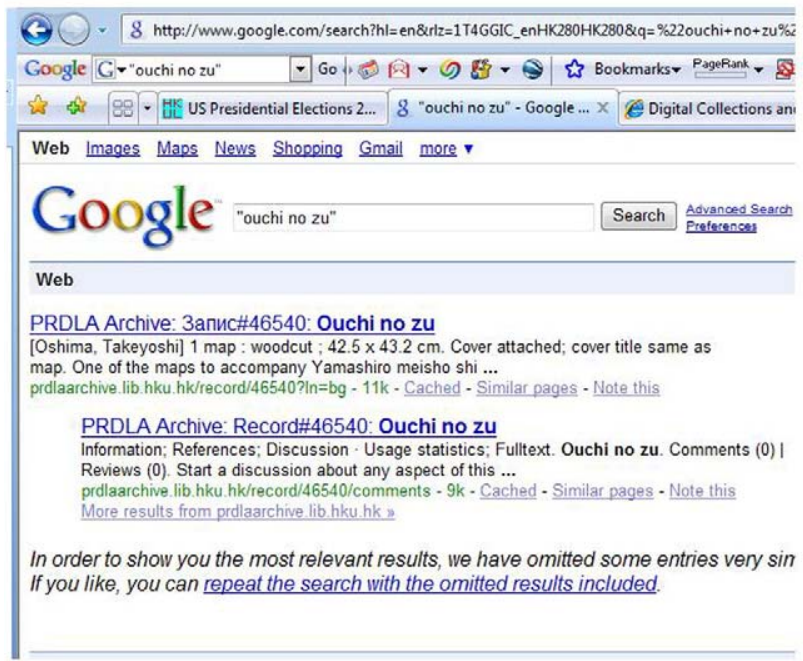

Figure 4. Google: "Ouchi no zu." 


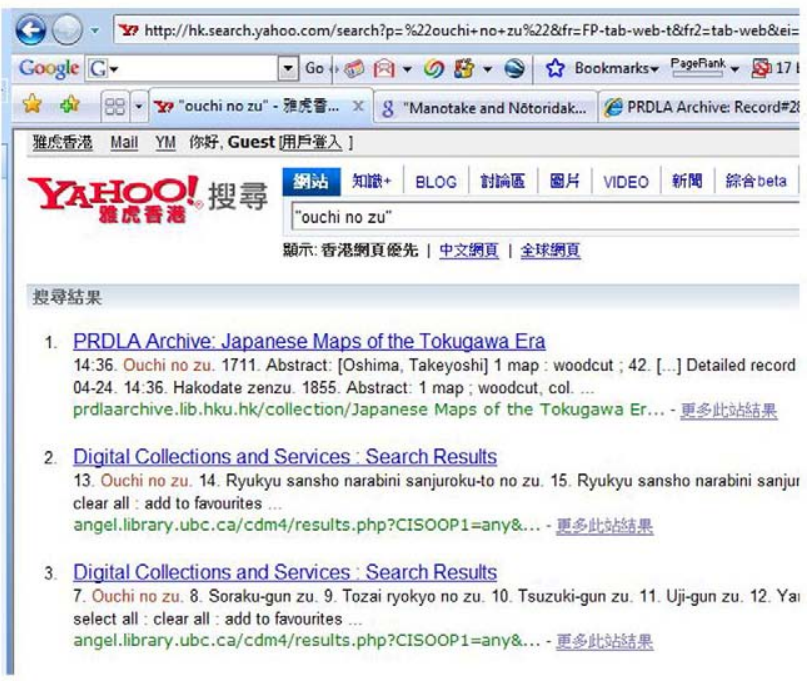

Figure 5. Yahoo: "Ouchi no zu."

shows PRL first and UW second. A search on "Ouchi no zu," a record from University of British Columbia, only shows the PRL entry in Google. Yahoo search results, however, show PRL first, followed by UBC, as shown in Figs. 4 and 5.

During the same week that HKU performed these searches, colleagues in Honolulu and London confirmed receiving the same results on the same searches.

These results demonstrate that PRL is linking users to those resources that may not have been discovered otherwise. In this manner, PRL is adding value to the original locally hosted databases that comprise PRL.

\section{The Deep Web}

Indexing or revealing the many hidden Web objects in the deep Web has long been a goal toward which many commercial and academic search engines continue to strive. Several reasons explain why these Web objects remain hidden. For example, to prevent undue traffic, some repositories use protocols to specifically exclude robots. Also, unless there are other links that point to these hidden Web objects, robots and crawlers will not find them. ${ }^{8}$

Most major search engines now provide procedures for repositories to follow in order to expose more of the deep Web to their robots. At one time, Google and Yahoo included OAI-PMH in these procedures; however, Google recently announced that they were retiring support for OAI-PMH in their sitemaps. ${ }^{9}$ This lack of support, therefore, shows the further value of PRL as a discovery source. PRL harvests OAI-PMH data from Web objects that may be hidden, in repositories that may not be completely visible to these search engines. PRL then exposes this harvested metadata to these search engines for their indexing. A recent study by the OAIster project indicated similar results. ${ }^{10}$
These search results, combined with the dissimilar nature of the 194 PRL collections, suggest that PRL's true value is as an indirect tool of 195 discovery. PRL has enabled the discovery of this hidden Web to 196 users of Google and Yahoo, and presumably pushed ranking of 197 these pages higher within these and other search engines. The 198 end-user will presumably find an item in the search engines, 199 bounce to PRL in Hong Kong, and then link to the originating 200 database in Hawaii, Wuhan, Singapore, or other PRDLA member 201 location, to begin a more focused search.

\section{The Future Value of PRL}

PRL has become a flagship project of PRDLA and serves to rally 204 member support and to promote their existence to the world. In 205 recognition, PRDLA has begun two new projects focused on PRL. 206 PRLDA is redesigning its logo and PRL graphics to enhance its 207 image. PRDLA has also created a collaboration for a new collection, 208 Oceania Digital Libraries (ODiL), expected for release in 2009.11 209

In summary, the PRDLA goal for PRL is the creation of a tool for 210 searching content about the Pacific Rim. However, the way that PRL 211 does this is not as a traditional destination database, but one that 212 acts as an intermediary between the search engines and the 213 originating database. Although this is beyond the original goal, the 214 PRDLA membership understands the value that PRL provides and 215 will continue to use this project and its Web pages to showcase 216 their collections.

\section{Notes}

1. “About [PRDLA]," http://www.prdla.ucmercedlibrary.info/?page_id=2, 219 (accessed March 30, 2009).

2. "Membership [of PRDLA]," http://prdla.ucmercedlibrary.info/about/ 221 membership/, (accessed March 30, 2009). 222

3. “OAI for Beginners," http://www.oaforum.org/tutorial/, (accessed March 30, 223 2009).

4. “Dublin Core Metadata Initiative," http://dublincore.org/usage/terms/dc/ 225 current-elements/, (accessed March 30, 2009).

5. "CDS Invenio Overview," http://cdsware.cern.ch/invenio/, (accessed March 227 30, 2009).

6. A "thank you" on this page to Dr. Ku Kam-ming of HKU, "CDS Invenio 229 Overview," http://cdsware.cern.ch/invenio/index.html, (accessed March 30, 230 2009).

7. "Sitemaps," http://en.wikipedia.org/wiki/Sitemaps, (accessed March 30, 232 2009).

8. Frank McCown, Michael L. Nelson, and Mohammad Zubair, "Search Engine 234 Coverage of the OAI-PMH Corpus," IEEE Internet Computing (March/April 235 2006), http://ieeexplore.ieee.org/stamp/stamp.jsp?arnumber $=01607990 . \quad 236$

9. John Mueller, "Retiring support for OAI-PMH in Sitemaps," Google Webmaster 237 Central Blog (April 23, 2008), http://googlewebmastercentral.blogspot.com/ 238 2008/04/retiring-support-for-oai-pmh-in.html, (accessed March 30, 2009). 239

10. Kat Hagedorn and Joshua Santelli, "Google Still Not Indexing Hidden Web 240 URLs," D-Lib Magazine 13, no.7/8 (2008), http://www.dlib.org/dlib/july08/ 241 hagedorn/07hagedorn.html, (accessed March 30, 2009).

11. “PRDLA," http://prdla.ucmercedlibrary.info/2008/10/oceania-digital- 243 libraries-odil-collaborative-digitization-project/, (accessed March 30, 2009). 244 